\title{
First study of small-cell 3D Silicon Pixel Detectors for the High Luminosity LHC
}

\author{
E. Currás ${ }^{1}$, J. Duarte-Campderrós ${ }^{1}$, M. Fernández ${ }^{1}$, A. \\ García $^{1}$, G. Gómez ${ }^{1}$, J. González ${ }^{1}$, R. Jaramillo ${ }^{1}$, D. Moya ${ }^{1}$, I. \\ Vila $^{1}$, S. Hidalgo ${ }^{2}$, M. Manna ${ }^{2}$, G. Pellegrini ${ }^{2}$, D. Quirion ${ }^{2}$, D. \\ Pitzl $^{3}$, A. Ebrahimi ${ }^{4}$, T. Rohe ${ }^{5}$, and S. Wiederkehr ${ }^{5}$ \\ ${ }^{1}$ Instituto de Físca de Cantabria, University of Cantabria / CSIC. \\ ${ }^{2}$ Instituto de Microelectrónica de Barcelona, Centro Nacional de \\ Microelectrónica. \\ ${ }^{3}$ Deutsches Elektronen Synchotron (DESY). \\ ${ }^{4}$ University of Hamburg. \\ ${ }^{5}$ Paul Scherrer Institut.
}

\begin{abstract}
A study of 3D pixel sensors of cell size $50 \mu m \times 50 \mu m$ fabricated at IMB-CNM using double-sided n-on-p 3D technology is presented. Sensors were bump-bonded to the ROC4SENS readout chip. For the first time in such a small-pitch hybrid assembly, the sensor response to ionizing radiation in a test beam of $5.6 \mathrm{GeV}$ electrons was studied. Results for non-irradiated sensors are presented, including efficiency, charge sharing, signal-to-noise, and resolution for different incidence angles.
\end{abstract}

\section{Introduction}

The LHC accelerator at CERN [1] will undergo a high luminosity upgrade, currently planned for the years 2023-2024, after which the instantaneous luminosity will reach $10^{35} \mathrm{~cm}^{-2} \mathrm{~s}^{-1}$ and the average number of hard protonproton scatters during any single proton bunch crossing (pileup) will exceed 
140. This will require upgraded detectors able to cope with extremely high track densities and to withstand unprecedented levels of radiation. In particular, vertex detectors must be tolerant to hadron fluences up to $2 \times 10^{16}$

$n_{e q} / \mathrm{cm}^{2}$, so detectors with a small charge carrier drift distance and a high electric field close to the collecting electrodes are required to minimize the impact of radiation-induced trapping. For a typical planar pixel sensor, this implies reducing as much as possible the sensor thickness. One possible choice of technology for the innermost layers, where the levels of radiation are expected to be highest, is 3D pixels, in which n-type columns are etched perpendicularly into a p-type bulk. Figure 1 shows a cross-section of such a device. With this arrangement of electrodes, charge carriers travel parallel to the sensor surface, and their travel distance is decoupled from the sensor thickness. For small pixel sizes, the sensors are intrinsically resistant to radiation and can be fully depleted at very low bias voltages owing to the small inter-electrode distance. Pixel sensors of very small pixel size are required to achieve a reasonably low detector occupancy. We present the first study of $3 \mathrm{D}$ pixels of cell size $50 \mu m \times 50 \mu m$ bump-bonded to a readout chip of matching pixel cell size.

\section{Experimental setup}

\subsection{Sensors and readout}

The sensors studied were fabricated at IMB-CNM using an n-on-p doublesided technology [2], where n-type (junction) columns are etched from the "top" side into a p-bulk, and p-type (ohmic) columns are etched from the "bottom" side. Columns have a diameter of $8 \mu \mathrm{m}$ and are not fully passing. The entire sensor has a thickness of $230 \mu m$, and the pixel cell size is $50 \mu m \times$ $50 \mu \mathrm{m}$. A p-stop ring surrounds each junction column to isolate the electrodes from accumulated surface charges. Figure 1 shows a sketch of the transverse cross-section (left) and a zoomed view of the layout (right) of such a sensor. Each sensor has a matrix of 155 columns $\times 160$ rows for a total of 24800 pixels. The sensors have a Ni/Au underbump metallization (UBM) deposited through an electroless process at CNM, and were bump-bonded to a frontend readout electronics called ROC4SENS [3] fabricated using a $250 \mathrm{~nm}$ CMOS process with radiation tolerant design. The ROC4SENS is a generic pixel readout chip (ROC) developed by the Paul Sherrer Institut (PSI) [4] 

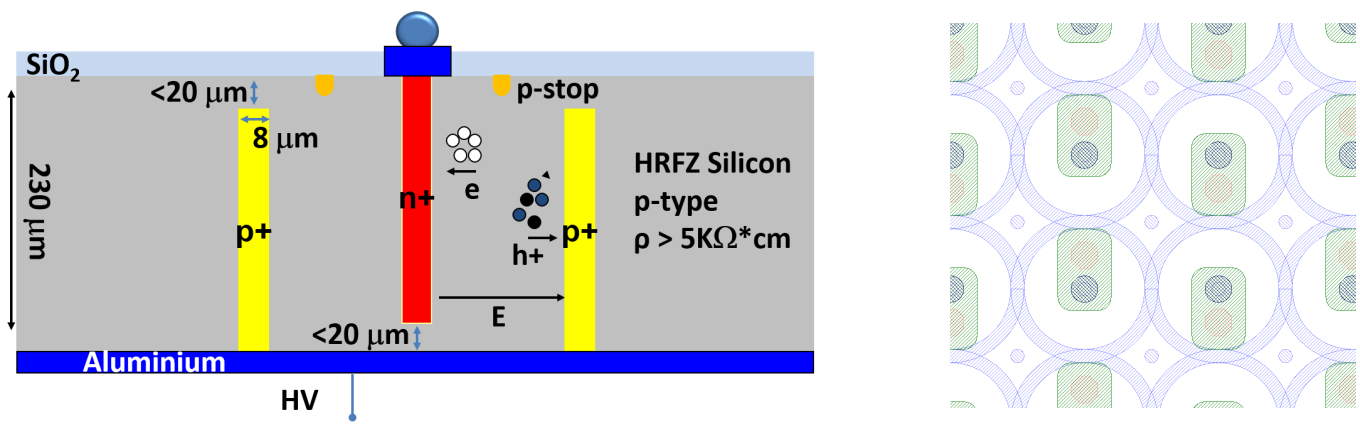

Figure 1: Left: cross-section of a double-sided 3D pixel sensor. Right: a zoomed view of the pixel sensor layout. The n-columns at the center of the p-stop rings are etched from the top side. The p-columns are etched from the bottom side.

specifically to characterize the sensor part of a hybrid pixel detector. In order not be limited to signals exceeding a certain threshold the chip has no discriminator for zero suppression. This in return means that the sampling time has to be defined by an external trigger (hold signal). After triggering the chip is insensitive to further signals until the hold signal is released after readout is finished. This dead time depends on the readout speed of the DAQ system and is not problematic for the application in test beams. In order to keep design and operation simple, no DACs have been implemented in the chip and four reference voltage have to be provided externally.

The single chip assembly (SCA) consisting of a sensor bump bonded to a R4S chip is glued and wire bonded to a dedicated printed circuit board (PCB) which is connected via a special interface to a so called digital test board (DTB) originally developed by PSI for the testing of the digital CMS pixel chip. In this paper we report on tests with 2 SCAs called SCA "A" and "B".

In order to study the electrical characteristics of 3D pixels of this cell size, small pad-like sensors were produced in the same wafers. These sensors contain only $100 \times 1003 \mathrm{D}$ pixels in which all electrodes (n-type columns) are connected together via an Aluminum metallization, but which are otherwise identical to the pixel cells previously described. Figure 2 shows the total dark current (left) and capacitance (right) as a function of reverse bias voltage for four of these pad-like sensors. Taking into account the fact that each pad sensor contains 10000 pixels, these figures show that, at a voltage of $20 \mathrm{~V}$ 

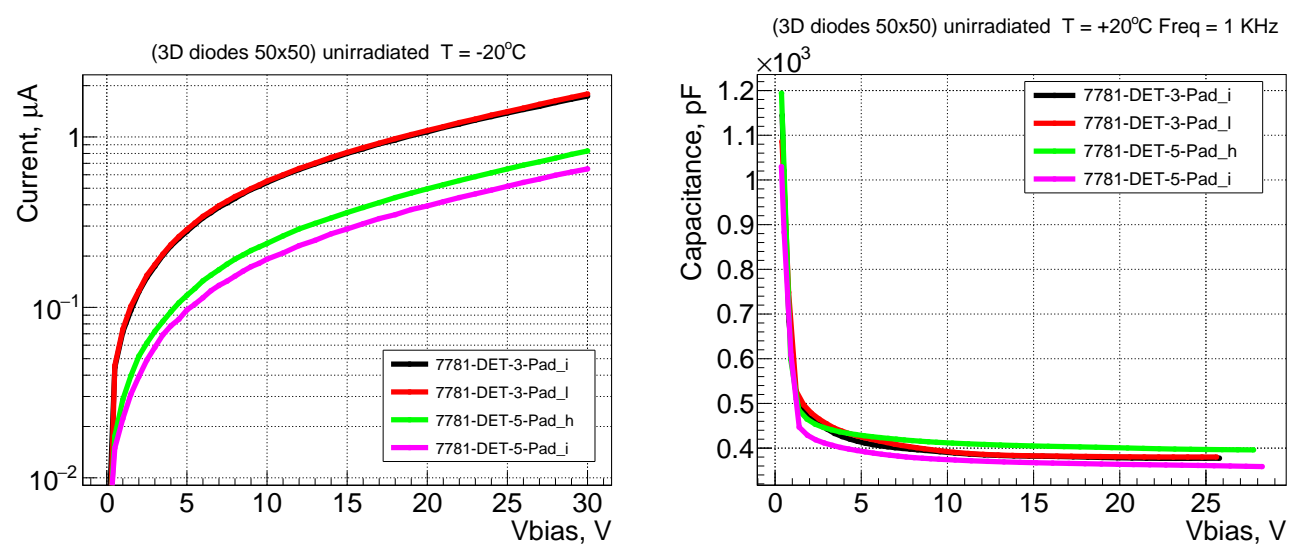

Figure 2: Electrical properties of small-pitch 3D pixels in pad-like sensors. Left: Total dark current as a function of reverse bias voltage. Right: Total capacitance as a function of reverese bias voltage.

and a temperature of $-20^{\circ} \mathrm{C}$, the dark current per pixel varies from 40 to 100 pA. The per-pixel capacitance is found to be about $40 \mathrm{fF}$, and the depletion voltage is about $10 \mathrm{~V}$, considerably lower than for typical planar pixel sensors.

\subsection{Test Beam Setup}

The sensor response to energetic minimum ionizing particles was studied at the DESY test beam facility [5], which provides a beam of electrons of energy in the range $1-6 \mathrm{GeV}$ adjustable through a spectrometer dipole magnet. The DESY synchrotron circulates a single electron bunch at a frequency of $1 \mathrm{MHz}$, and the particle rate in the test beam depends on beam line, energy, collimator settings, target material and operation. For the study presented here, an energy of $5.6 \mathrm{GeV}$ was used and particle rates of about $40 \mathrm{kHz}$ were achieved. Tracking was provided by the DATURA beam telescope [6], which consists of six planes of MIMOSA26 pixel detectors of pitch $18.4 \mu \mathrm{m} \times 18.4 \mu \mathrm{m}$, with an intrinsic telescope plane resolution of about 3.2 $\mu m$ in the best possible conditions of particle energy, scattering and distance between planes [7]. A trigger logic unit (TLU) [8] requires coincidence of two trigger input signals generated by a pairs of PMT-scintillator assemblies placed upstream of the telescope, forming a trigger window of approximately $1 \mathrm{~cm} \times 1 \mathrm{~cm}$, slightly larger than the device under test (DUT). The DUT is 


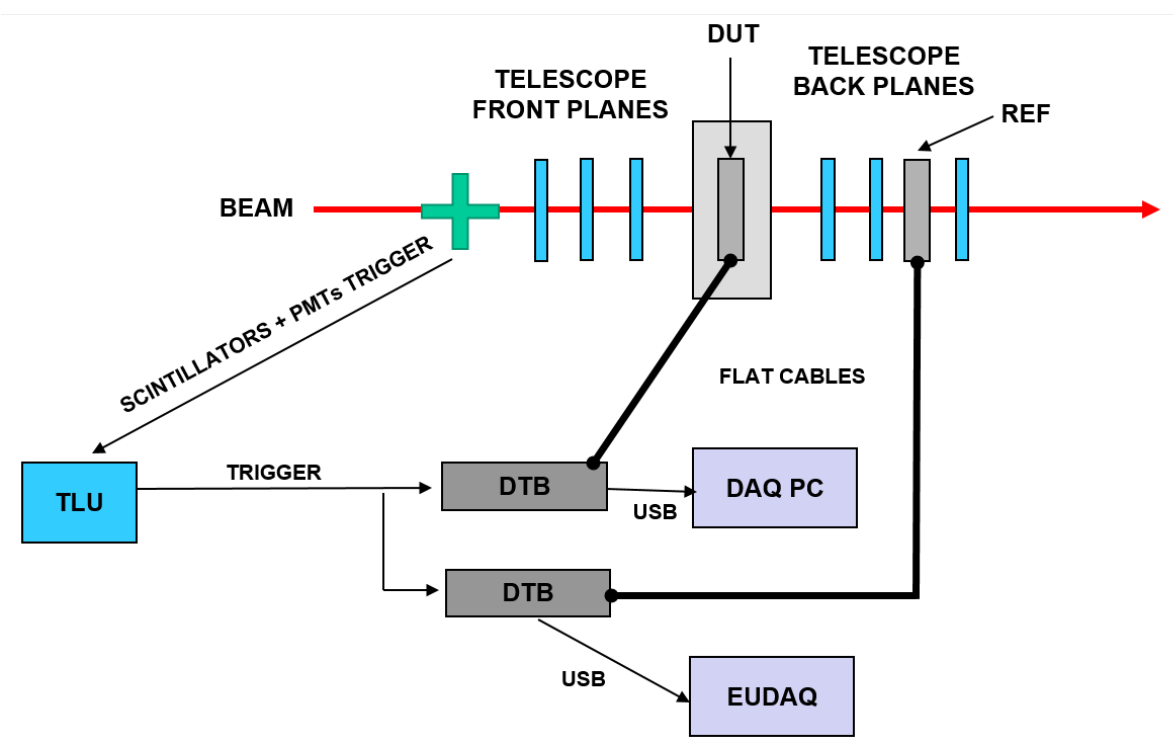

Figure 3: Sketch of test beam setup.

placed in the middle of the telescope, sandwiched by two triplets of telescope planes. The sensor mother board is mounted on a motorized platform which allows to remotely adjust the position and the inclination of the sensor with respect to the beam. The MIMOSA sensors operate in a rolling shutter mode with an integration time of approximately $120 \mu$ s, which causes track pile-up. A SCA with pitch $100 \mu m \times 150 \mu m$ equipped with a fast PSI46V2.1dig chip, as used in the outer layersof the CMS Phase-1 upgrade pixel detector, is used as timing reference to tag the track on the DUT. It is also read out with a DTB. Figure 3 shows a schematic diagram of the test beam setup.

\section{Data reconstruction}

Data from the telescope planes and the reference sensor are stored via specialized producers by the EUDAQ data acquisition framework [9]. Data from the DUT (ROC4SENS), not yet integrated into EUDAQ, was pre-processed by the digital test board (DTB) with a customized firmware and stored via USB on a separate data acquisition PC. These two data streams share the same TLU trigger, and are fed into an analysis software which integrates all information and performs an offline event reconstruction. 


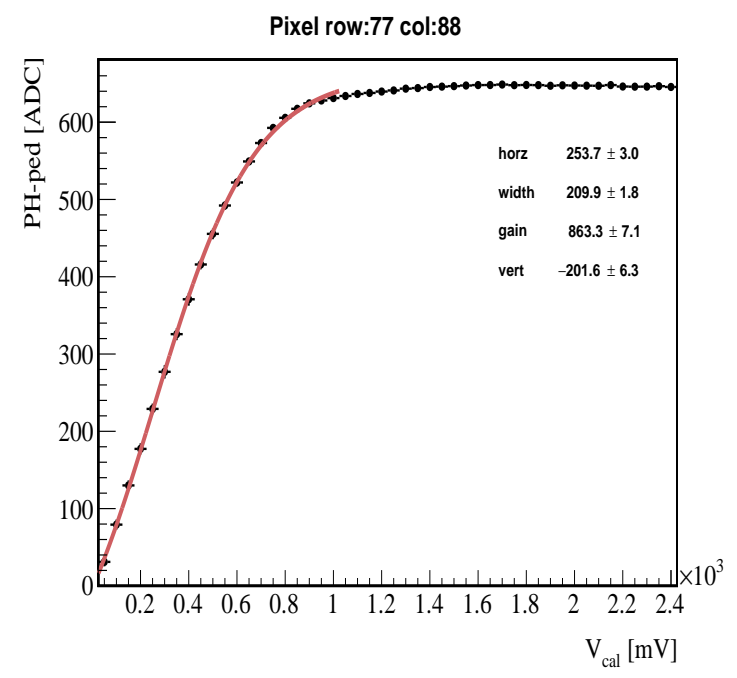

Figure 4: Example of calibration for one single ROC pixel. The pulse height is recorded (in units of ADC) as a function of an applied calibration voltage (Vcal, in $\mathrm{mV}$ ). The fit is then used to obtain the gain of each ROC pixel.

A calibration of each pixel of the readout chip is performed by injecting a calibration pulse. Figure 4 shows the calibration for one single ROC pixel, where the pedestal-subtracted pulse height in units of ADC is recorded as a function of the calibration signal amplitude (Vcal). A Fermi function is fit to these distributions and applied offline to each pixel separately to properly handle relative pixel-to-pixel gain variations.

Once the data taking begins, the DTB uses the first 100 readouts to determine the pedestal of each pixel. Figure 5 shows the average pedestal value (in ADC counts) recorded for each pixel. The column pattern is a known ROC feature which arises from gyrator circuit variations at the end of each column. During a run the pedestal subtracted pulse height $(\Delta P H)$ is calculated for each pixel. The difference between neighbouring pixels is used to define if a pixel was hit, with a threshold value of $24 \mathrm{ADC}$ counts. A region of interest (ROI) of 7 rows times 5 columns around the hit pixel is stored for off line processing. This ROI technique is used to reduce the amount of data, as there are limits in the memory and data transfer of the DTB. Hits are therefore defined based on a relative signal threshold and not on a signalto-noise cut. The noise, calculated as the width of the $\Delta P H$ distribution, is approximately 3 ADC counts, as shown in Figure 6. Offline, a column-wise 


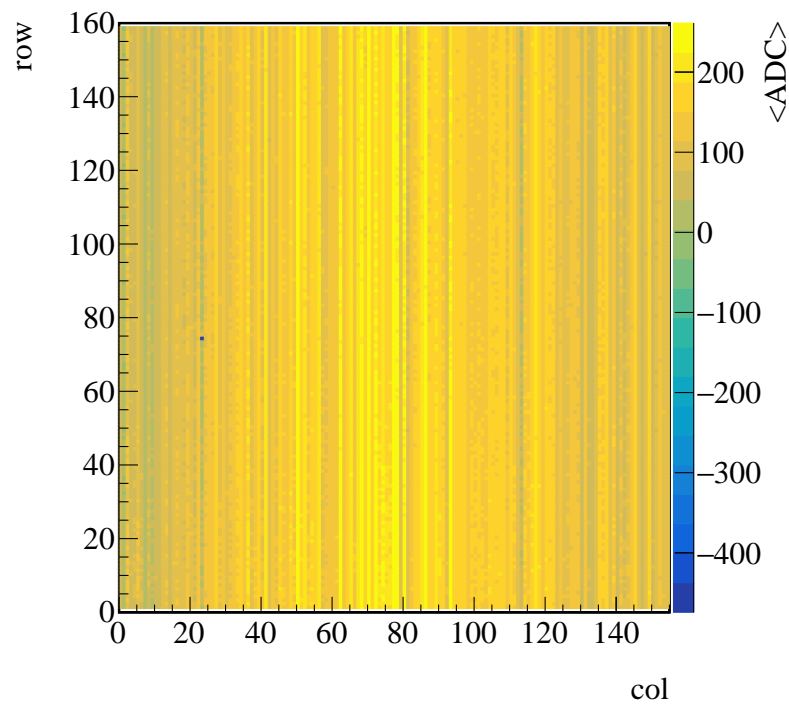

Figure 5: Pedestal map. Average pedestal for each pixel in ADC counts.

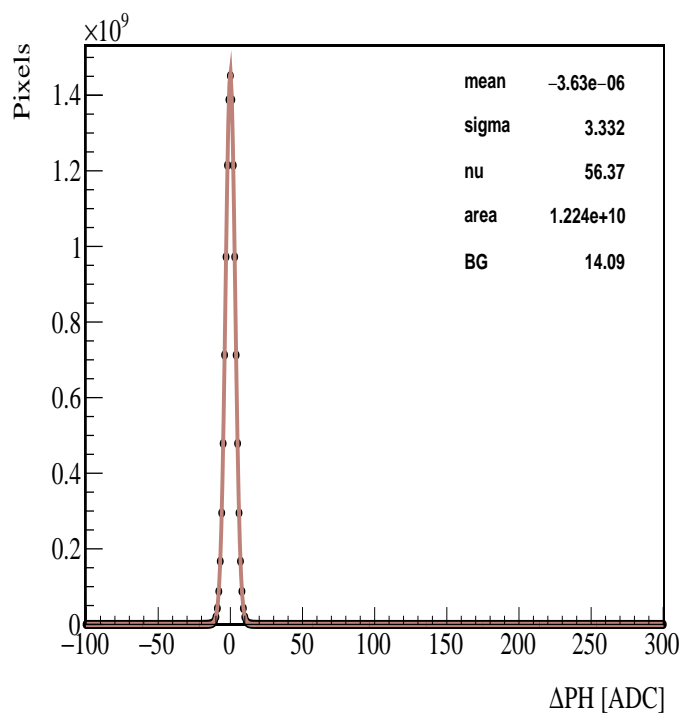

Figure 6: Pedestal-subtracted pulse height for all pixels in units of ADC counts. A t-Student function is fit to the distribution. The sigma parameter of the fit provides an estimate of the ROC noise. 
common mode correction is applied for each pixel in the ROI by subtracting the nearest edge pixel in each of the 5 columns to in-between pixels. For the corrected pixels, pedestal-subtracted pulse heights are recalculated, and hits are re-defined by requiring a difference greater than 10 ADC counts with respect to neighboring pixels. Finally, all neighboring hits in rows and columns are clustered, and the calibration Fermi functions are applied to each pixel to obtain a uniform charge response.

The calibrated charge obtained is normalilzed to the expected average charge deposition of 76 electrons per micron for a MIP traversing an unirradiated sensor, since the equivalence between charge and voltage for the calibration pulse of the ROC4SENS is not well known.

\section{Results}

The sensors were operated at room temperature and at a bias voltage of $25 \mathrm{~V}$, safely above the full depletion voltage obtained from CV measurements (below $10 \mathrm{~V}$, see Figure 2). Figure 7 shows the hit map for sensor A at perpendicular incidence. All $155 \times 160$ pixels are shown. Apart from the noisy regions which are due to a bad UBM deposition, the sensor shows a large area of uniform behavior which is more than acceptable for characterization purposes.

The track incidence angle is measured with respect to the direction perpendicular to the sensor. High statistics samples were recorded for both sensors $\mathrm{A}$ and $\mathrm{B}$ at perpendicular track incidence $\left(0^{\circ}\right)$ and at $12^{\circ}$, the arctangent of the sensor pitch to width ratio. Figure 8 shows the cluster size distribution in number of cluster columns (sensor X direction) and rows (sensor $\mathrm{Y}$ direction). The sensor was oriented such that it rotated around its $\mathrm{X}$-axis, and therefore the number of cluster columns peaks at 1 regardless of the angle of incidence, while the most likely number of cluster rows clearly increases with sensor inclination.

\subsection{Charge}

Figure 9 shows the calibrated charge distribution for clusters matched to a track for sensor A at perpendicular incidence. Recall that the charge is normalized to the expected MIP charge deposition of 76 electrons per micron, or 17 kilo electrons for a $230 \mu m$ thick sensor. The charge collection can be 


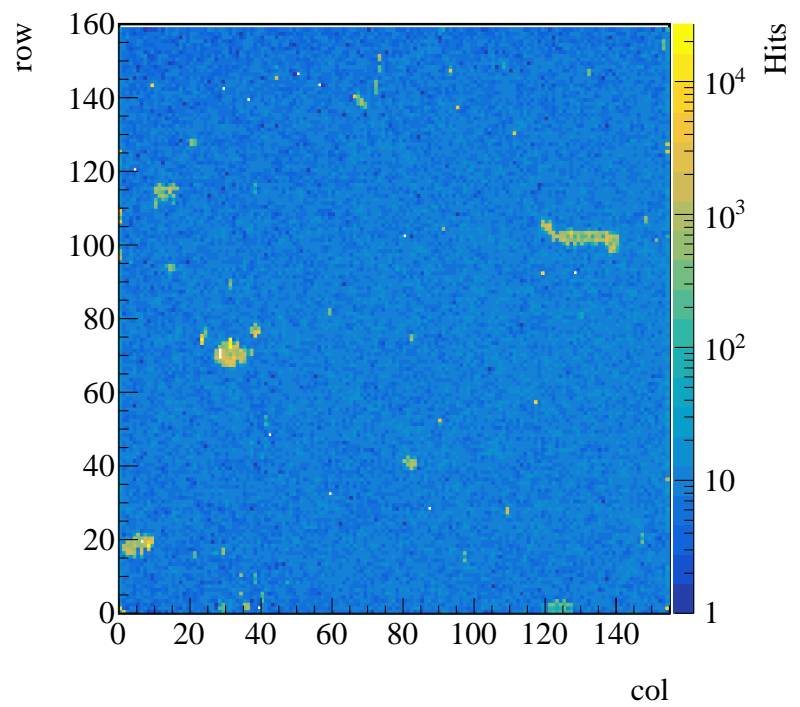

Figure 7: Hit map for sensor A at perpendicular incidence. All 24800 pixels are shown. Noisy regions from bad UBM deposition are masked out from the analysis.
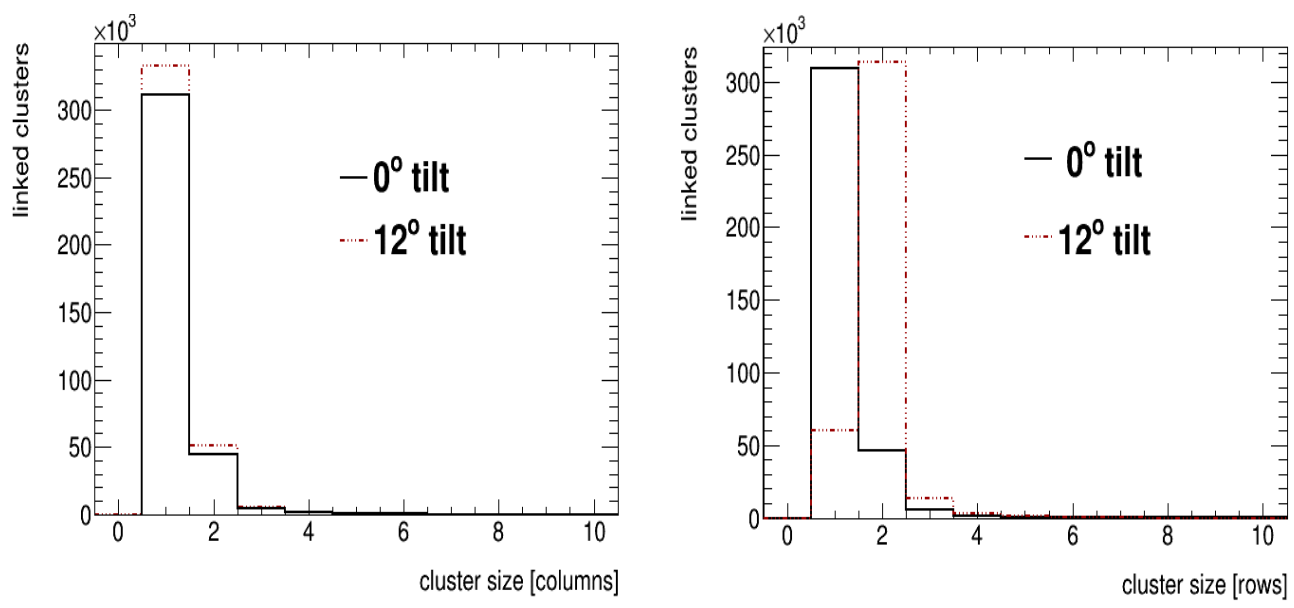

Figure 8: Distribution of the number of cluster columns (left) and rows (right) for perpendicular and for $12^{\circ}$ incidence.

studied as a function of pixel cell position. Figure 10 shows the cluster charge as a function of track position for sensors A and B at perpendicular incidence 


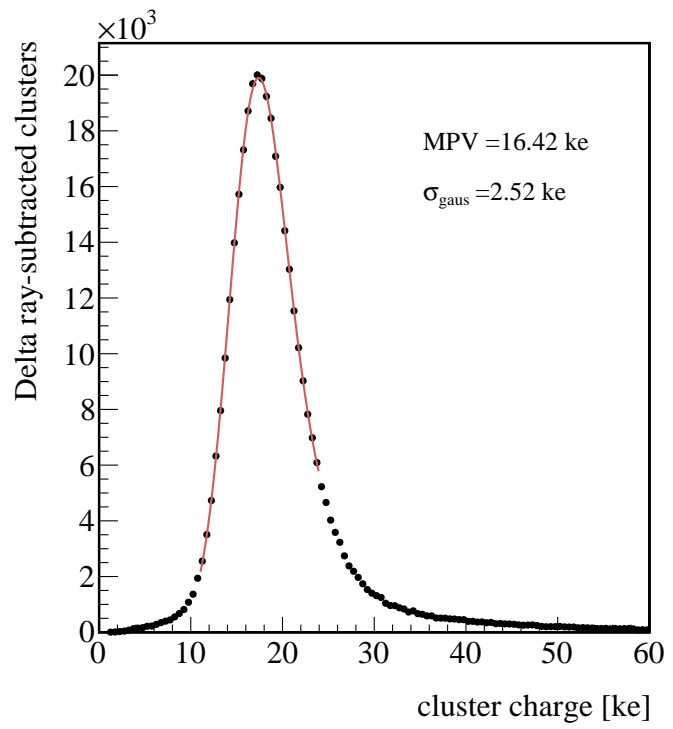

Figure 9: Cluster charge for clusters matched to a track. The charge is normalized to peak at 76 electrons per micron, or 17 kilo electrons for a 230 $\mu m$ thick sensor.

in an array of $2 \times 2$ pixel cells. It is important to remark that in this figure, and in all subsequent figures showing a four-pixel array, information from all such (good) pixel arrays from the entire sensor is mapped together into a single pixel array in order to obtain reasonably high statistics. The $x$ and $y$ axis in these figures are the track coordinates at the plane of the DUT modulo $100 \mu \mathrm{m}$. At perpendicular incidence, the pixel cell structure can be deduced from the regions of lower charge collection, which correspond to the positions of the union and ohmic columns. The structure observed in this figure (and similarly in the efficiency figures below) should be compared to the structure of Figure 1 (right) delimited by the nine ohmic columns shown, defining a 2x2 pixel array. The charge collection becomes uniform along the pixel cell for non-perpendicular incidence. Figure 11 shows the corresponding charge distributions for $12^{\circ}$ incidence.

\section{$4.2 \quad$ Efficiency}

The hit detection efficiency is defined as the fraction of telescope tracks matched to the reference sensor which also show a hit matched to the DUT. 

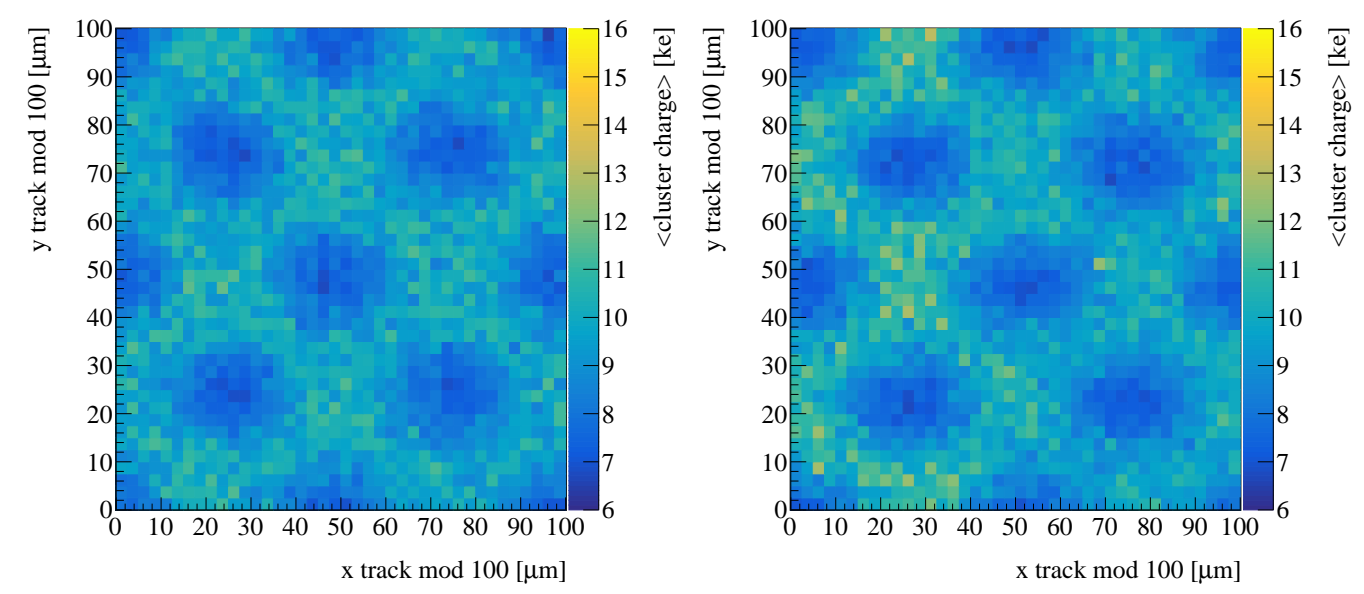

Figure 10: Cluster charge at perpendicular incidence as a function of track position in an array of $2 \times 2$ pixels for sensor A (left) and B (right).
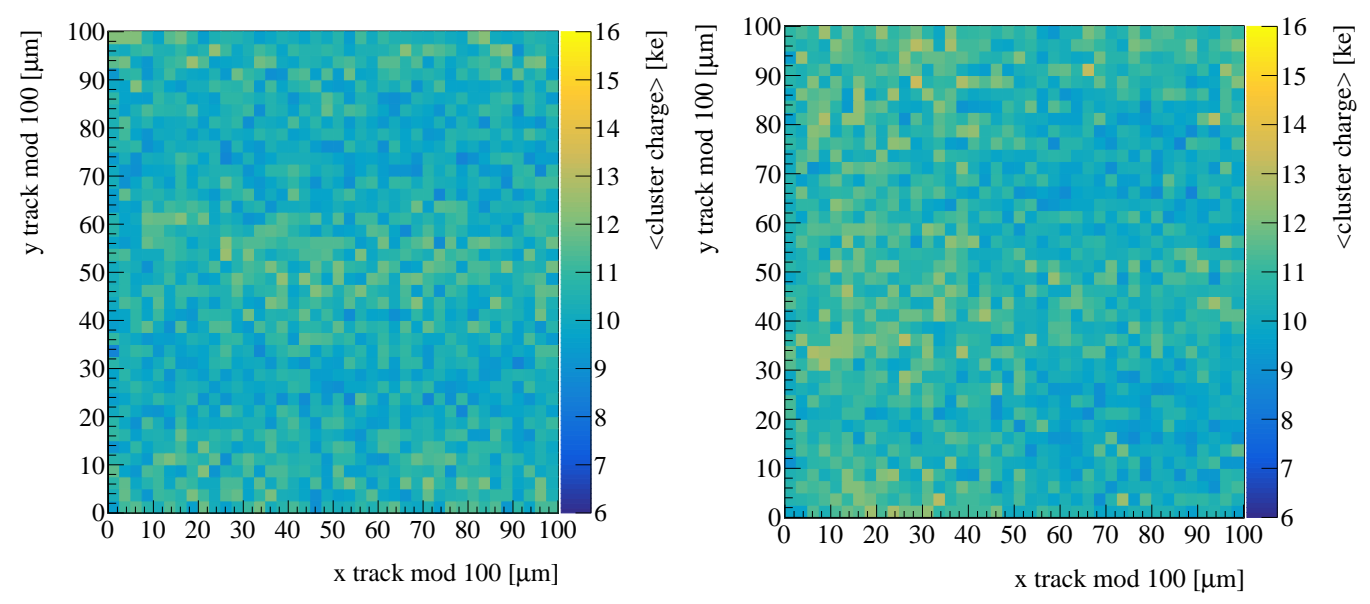

Figure 11: Cluster charge at $12^{\circ}$ incidence as a function of track position in an array of $2 \times 2$ pixels for sensor A (left) and B (right). 
Telescope tracks are selected by requiring hits on all six planes of the telescope. In addition, two tracks are formed by using the set of three planes before and after the DUT. The distance between the position of these two tracks extrapolated to the plane of the DUT is required to be smaller than $100 \mu \mathrm{m}$. A track is matched to the reference sensor if there is a hit in the sensor at a distance smaller than twice its binary resolution. Matched tracks are also required to be isolated by imposing the condition that no additional tracks be found within a radius of $300 \mu \mathrm{m}$ from the matching hit. Finally, tracks passing these selection criteria are matched to the DUT if a DUT his is found at a distance smaller than 6 times its binary resolution from the track projection at the DUT plane. The hit definition uses a threshold of $\triangle P H>24 \mathrm{ADC}$ counts compared to the neighbouring pixels as discussed in Sec. 3. Figure 12 shows the sensor efficiency at perpendicular incidence for both sensors as a function of track position in an array of $2 \times 2$ pixel cells. At perpendicular incidence, the efficiency is very close to $100 \%$ except in the regions of the union (n-type) columns, where it drops to approximately $94 \%$, and the ohmic columns (p-type) where it is approximately $97 \%$. This slight loss of efficiency is recovered when tilting the sensor. Figure 13 shows the corresponding efficiency for an incidence angle of $12^{\circ}$, where it can be seen that the efficiency is essentially constant and close to $100 \%$ throughout the sensor.
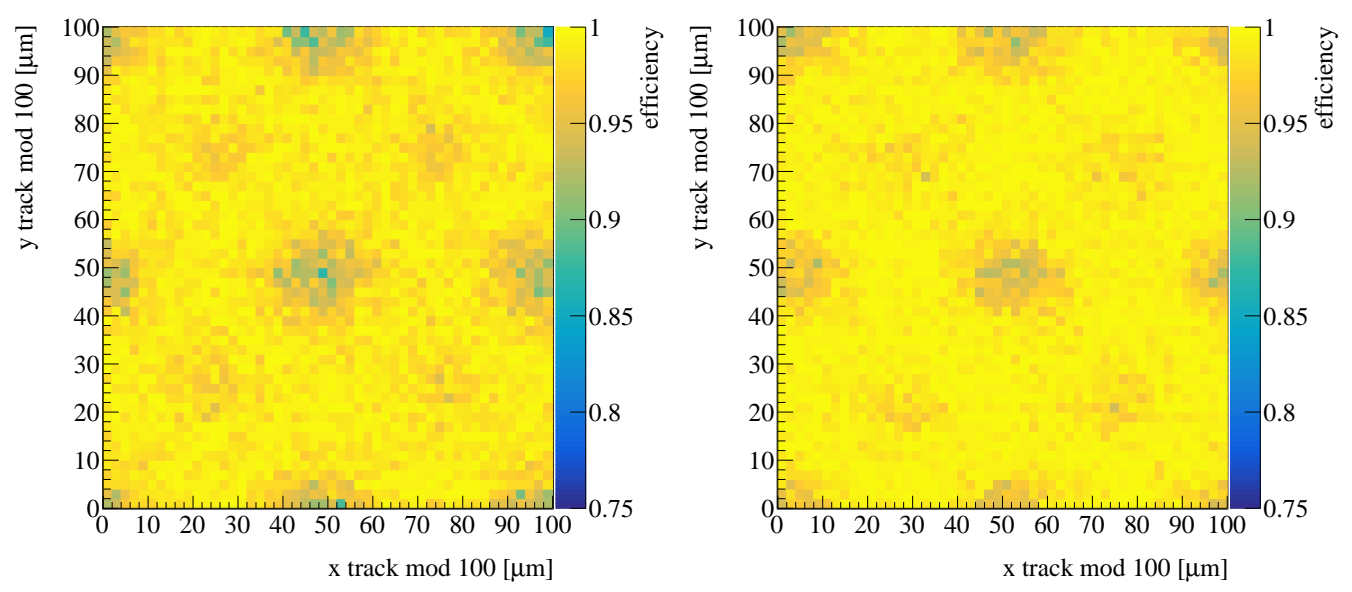

Figure 12: Sensor tracking efficiency at perpendicular incidence as a function of track position in an array of $2 \times 2$ pixels for sensor A (left) and B (right). 

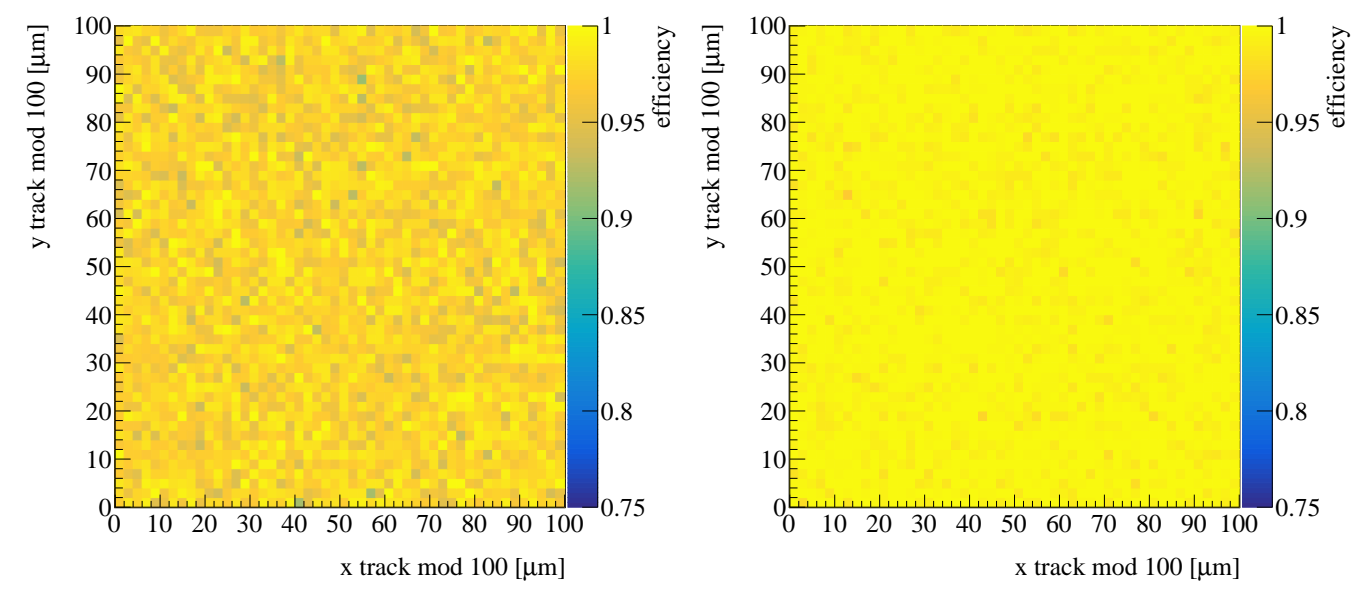

Figure 13: Sensor tracking efficiency at $12^{\circ}$ incidence as a function of track position in an array of $2 \times 2$ pixels for sensor A (left) and B (right).

The overall efficiency, integrated over the entire sensor, was studied as a function of incidence angle for sensor A. The results are summarized in Figure 14. For incidence angles above $5^{\circ}$, the overall efficiency remains above $99.5 \%$.

\subsection{Charge sharing}

The sharing of pixel cluster charge between adjacent pixels helps to improve the spatial resolution with respect to the binary resolution. Charge sharing is expected to be largest near pixel boundaries. Figure 15 shows the mean cluster size at perpendicular incidence. As expected, the mean cluster size is close to unity near the pixel cell centers, which are dominated by singlepixel clusters. The mean cluster size rises significantly above 1 near pixel boundaries where clusters of 2 and 3 pixels make a significant contribution. Figure 16 shows the corresponding mean cluster size for $12^{\circ}$ incidence angle. As expected, the mean cluster size increases significantly over the entire sensor (notice the change of scale), and the region of lower charge sharing shrinks closer to the pixel cell center along the tilted direction (Y). 


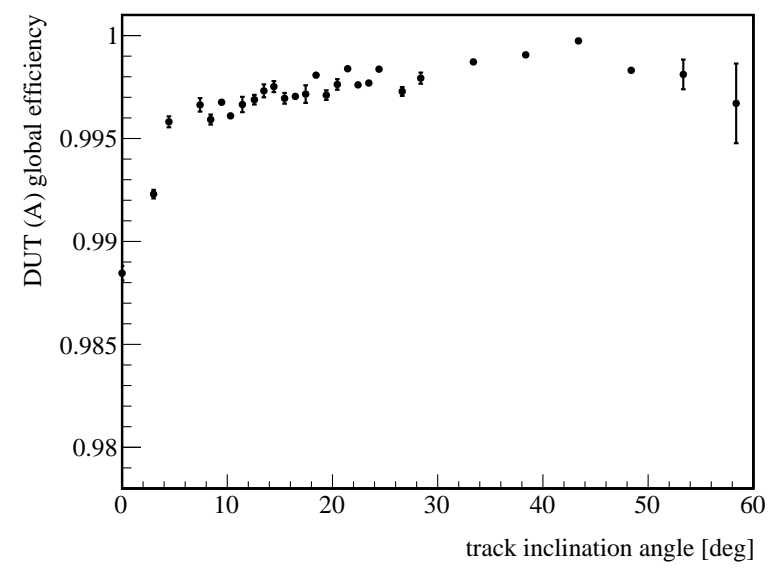

Figure 14: Overall sensor efficiency as a function of track incidence angle
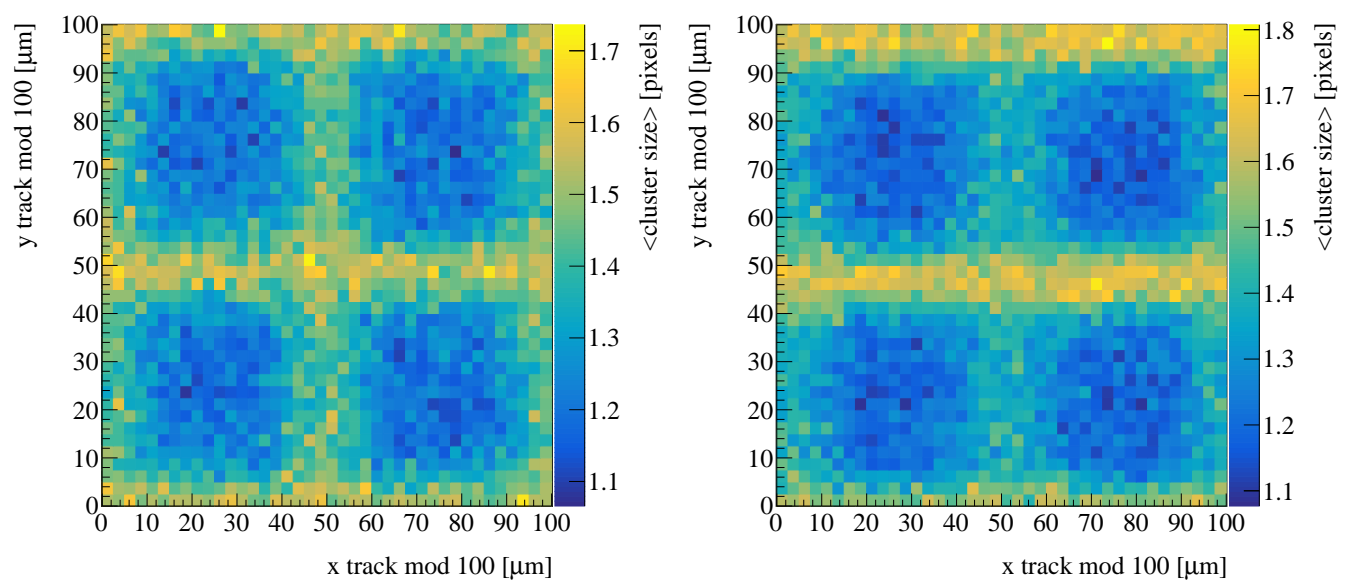

Figure 15: Mean cluster size at perpendicular incidence as a function of track position in an array of $2 \times 2$ pixels for sensor A (left) and B (right). 

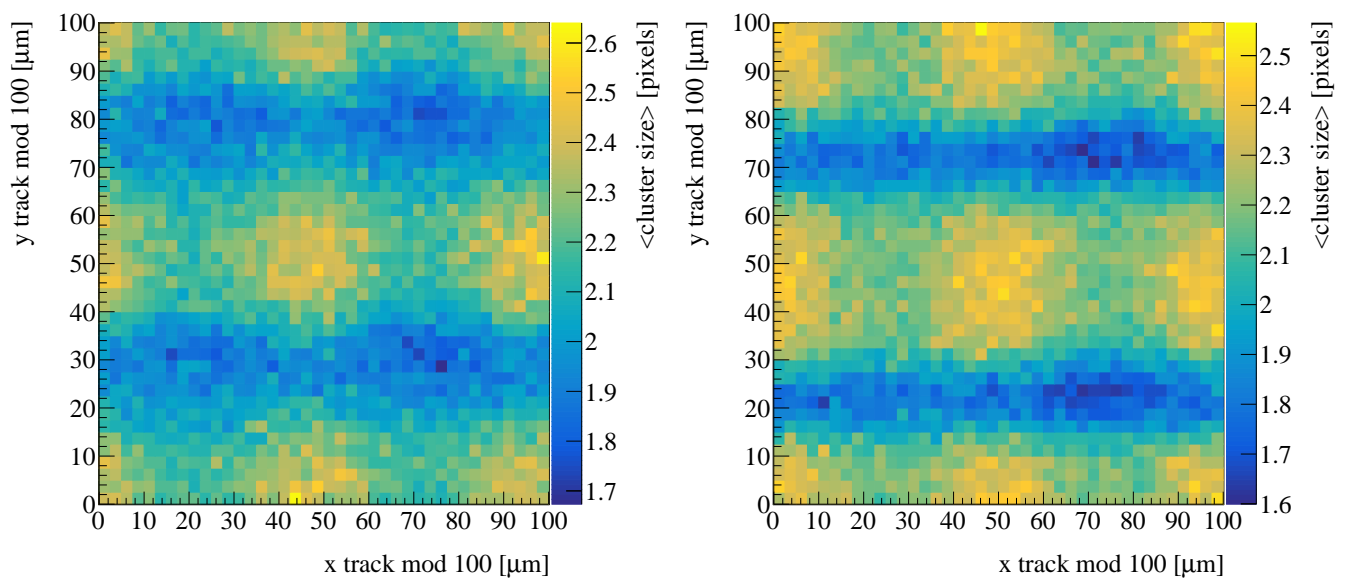

Figure 16: Mean cluster size at $12^{\circ}$ incidence as a function of track position in an array of $2 \times 2$ pixels for sensor $A$ (left) and B (right).

\subsection{Spatial resolution}

In order to determine the sensor resolution, the DUT is placed as close as physically possible to the upstream telescope triplet. The triplet track is extrapolated to the plane of the DUT taking into account multiple scattering by using the General Broken Lines (GBL) formalism [10] and the distance to the DUT hit position is computed. The width of this residual distribution has a contribution from the telescope resolution, which is known, and from the intrinsic DUT resolution, which is therefore calculated as:

$$
\sigma_{D U T}=\sqrt{\left(\sigma_{\Delta y}\right)^{2}-\left(\sigma_{\text {telescope }}\right)^{2}}
$$

Only DUT clusters matched to a telescope track and to the reference sensor are used. In addition, only clusters which have a charge close to the Landau most probable value are used. This cut in charge rejects delta rays produced in the sensor which would severely affect the position resolution, since delta electrons produced at the point of incidence typically travel a long distance inside the sensor, producing very large clusters of large charge and with a centroid far away from the track incidence point. Figure 17 shows the residual width as a function of cluster charge, where the deterioration at large charge due to delta rays can be seen.

The optimal DUT resolution is calculated using clusters from $12^{\circ}$ incidence tracks after rejecting delta rays. The residual distribution between the 


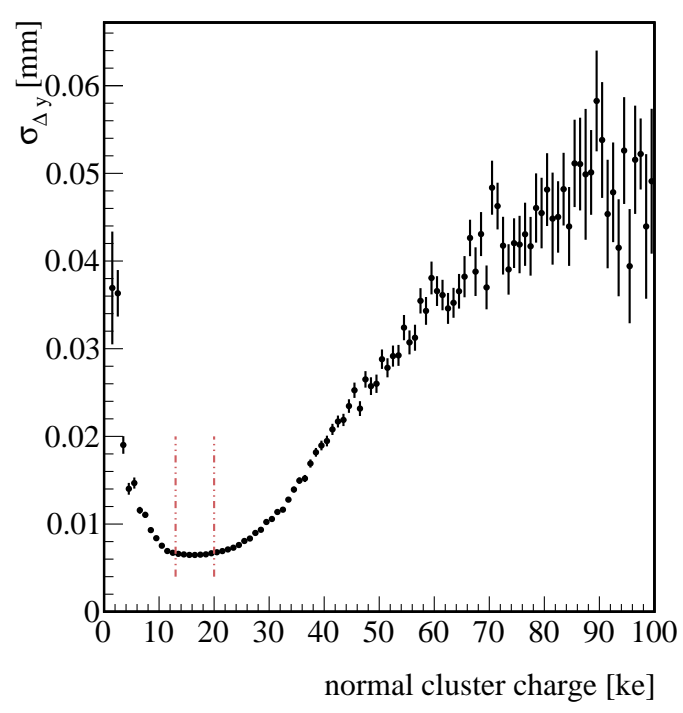

Figure 17: Residual width as a function of cluster charge. Only clusters with charge in the region inside the dashed lines are used for the DUT resolution measurement. The resolution deterioration at large charge is due to the influence of delta rays. 
telescope track projection and the cluster center has a width of $6.46 \mu \mathrm{m}$, as shown in Figure 18. After subtracting in quadrature the telescope resolution evaluated with the GBL formalism, the DUT resolution at $12^{\circ}$ incidence is found to be $3 \mu \mathrm{m}$.

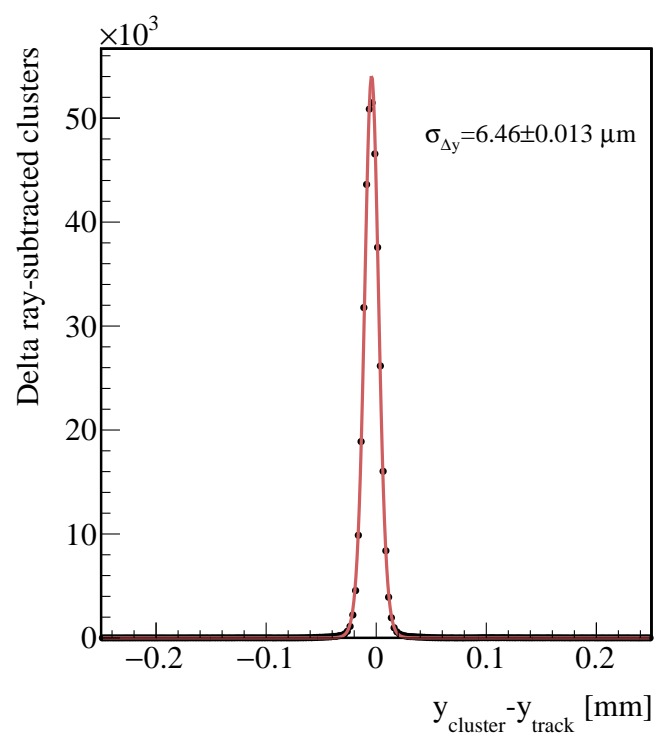

Figure 18: Residual distribution for $12^{\circ}$ incidence for clusters matched to a telescope track after delta ray rejection. The width has a contribution from the telescope resolution and from the DUT resolution.

The resolution has been studied as a function of incidence angle. The resolution is expected to be close to the binary resolution at perpendicular incidence $(14.4 \mu \mathrm{m}$ for a pitch of $50 \mu \mathrm{m})$, and improve with charge sharing as the cluster size increases, reaching an optimal value at the arc-tangent of the pitch to thickness ratio $\left(12^{\circ}\right.$ for these sensors). Figure 19 shows the DUT resolution as a function of incidence angle.

\section{Conclusions}

For the first time, small-pitch 3D pixel sensors of cell size $50 \mu m \times 50 \mu m$ have been characterized using a small-pitch readout chip, the ROC4SENS. The sensor response to minimum ionizing radiation was studied at a test beam of $5.6 \mathrm{GeV}$ electrons at DESY. The sensors show good performance in terms of 


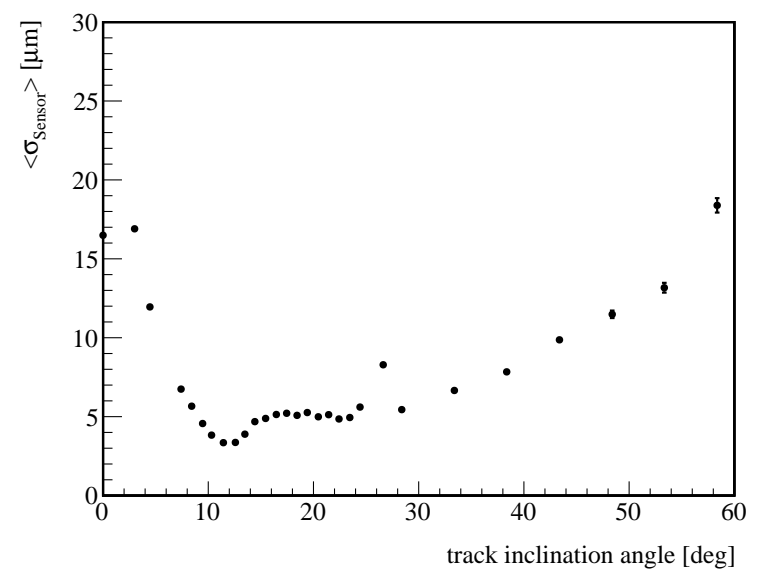

Figure 19: DUT resolution as a function of track incidence angle.

efficiency, charge sharing, and hit position resolution. For incidence angles larger than 5 degrees (with respect to perpendicular incidence), the single hit efficiency is found to be larger than $99.5 \%$, and a hit position resolution between 3 and $4 \mu \mathrm{m}$ at optimal incidence angle is achieved. The unirradiated sensors were operated at $25 \mathrm{~V}$, safely above the full depletion voltage which is below $10 \mathrm{~V}$, yet considerably lower than typical planar sensor operating voltages. In a future characterization, we plan to irradiate these sensors with protons to assess their robustness to irradiation and therefore their adequacy for the high luminosity upgrades of the LHC vertex detectors. A recent study [11] of small pitch 3D pixels irradiated up to a fluence of $3 \times 10^{16}$ $n_{e q} / \mathrm{cm}^{2}$ shows that the 3D technology is suitable for the inner layers of HL-LHC vertex detectors.

\section{Acknowledgments}

This project has received funding from the European Unions Horizon 2020 research and innovation programme under grant agreement No 654168, and from the Spanish MINECO ministry, under grants FPA2014-55295-C3-1-R and FPA2015-71292-C2-1-P. We thank DESY for their test-beam infrastructure and support, and the PSI team involved in the ROC4SENS development. 


\section{References}

[1] P. B. L. Evans, Lhc machine, JINST 3 (S08001). doi:10.1088/17480221/3/08/S08001.

[2] G. Pellegrini, J. Balbuena, D. Bassignana, E. Cabruja, C. Fleta, C. Guardiola, M. Lozano, D. Quirion, M. Ulln, 3D double sided detector fabrication at IMB-CNM, Nuclear Instruments and Methods in Physics Research Section A 699 (2013) 27 - 30, Proceedings of the 8th International Hiroshima Symposium on the Development and Application of Semiconductor Tracking Detectors. doi:https://doi.org/10.1016/j.nima.2012.05.087.

URL http://www.sciencedirect.com/science/article/pii/ S0168900212006092

[3] T. Rohe, ROC4SENS - a generic readout chip for sensor studies, https://indico.cern.ch/event/663851/contributions/2788211 [Accessed: 2016.06.01] (2017).

[4] Paul Sherrer Institut, https://www.psi.ch [Accessed: 2018.06.01].

[5] DESY, http://www.desy.de [Accessed: 2018.06.01].

[6] DATURA - EUDET-type beam telescopes, https://telescopes. desy.de/DATURA [Accessed: 2018.06.01].

[7] H. Jansen, Resolution studies with the DATURA beam telescope, JINST 11 (12) (C12031).

[8] D. Cussans, Description of the JRA1 Trigger Logic Unit (TLU), v0.2c, Tech. Rep. EUDET-Memo-2009-4, http://www.eudet.org/e26/e28/ e42441/e57298/EUDET-MEMO-2009-04.pdf [Accessed: 2018.06.01].

[9] EUDAQ Software Developers, http://eudaq.github.io, [Accessed: 2018.06.01].

[10] C. Kleinwort, General broken lines as advanced track fitting method, Nuclear Instruments and Methods in Physics Research Section A 673 (2012) 107 -110, http://www.sciencedirect.com/science/article/ pii/S0168900212000642. doi:https://doi.org/10.1016/j.nima. 2012.01 .024$. 
[11] J. Lange, G. Giannini, S. Grinstein, M. Manna, G. Pellegrini, D. Quirion, S. Terzo, D. V. Furelos, Radiation hardness of small-pitch 3D pixel sensors up to a fluence of $3 \mathrm{e} 16 \mathrm{neq} / \mathrm{cm} 2$, https ://arxiv.org/ abs/1805.10208 [Accessed: 2018.06.01] (2018). 